\title{
Vildagliptin reduces cardiac ischemic-reperfusion injury in obese orchiectomized rats
}

\author{
Wanpitak Pongkan1,2,3, Hiranya Pintana1,2, Thidarat Jaiwongkam 1,2,3, \\ Sasiwan Kredphoo 1,2,3, Sivaporn Sivasinprasasn 1,2,3, Siriporn C Chattipakorn 1,2,4 and \\ Nipon Chattipakorn'1,2,3 \\ ${ }^{1}$ Cardiac Electrophysiology Research and Training Center, Faculty of Medicine, Chiang Mai University, \\ Chiang Mai, Thailand \\ ${ }^{2}$ Cardiac Electrophysiology Unit, Department of Physiology, Faculty of Medicine, Chiang Mai University, \\ Chiang Mai, Thailand \\ ${ }^{3}$ Center of Excellence in Cardiac Electrophysiology Research, Chiang Mai University, Chiang Mai, Thailand \\ ${ }^{4}$ Department of Oral Biology and Diagnostic Science, Faculty of Dentistry, Chiang Mai University, \\ Chiang Mai, Thailand
}

Correspondence should be addressed to $\mathrm{N}$ Chattipakorn Email nchattip@gmail.com

\begin{abstract}
Obesity and testosterone deprivation are associated with coronary artery disease. Testosterone and vildagliptin (dipeptidyl peptidase-4 inhibitors) exert cardioprotection during ischemic-reperfusion (I/R) injury. However, the effect of these drugs on $I / R$ heart in a testosterone-deprived, obese, insulin-resistant model is unclear. This study investigated the effects of testosterone and vildagliptin on cardiac function, arrhythmias and the infarct size in I/R heart of testosterone-deprived rats with obese insulin resistance. Orchiectomized $(\mathrm{O})$ or sham operated $(\mathrm{S})$ male Wistar rats were divided into 2 groups to receive normal diet (ND) or high-fat diet (HFD) for 12 weeks. Orchiectomized rats in each diet were divided to receive testosterone ( $2 \mathrm{mg} / \mathrm{kg}$ ), vildagliptin $(3 \mathrm{mg} / \mathrm{kg}$ ) or the vehicle daily for 4 weeks. Then, $1 / \mathrm{R}$ was performed by a 30-min left anterior descending coronary artery ligation, followed by a 120-min reperfusion. LV function, arrhythmia scores, infarct size and cardiac mitochondrial function were determined. HFD groups developed insulin resistance at week 12. At week 16, cardiac function was impaired in NDO, HFO and HFS rats, but was restored in all testosterone- and vildagliptin-treated rats. During I/R injury, arrhythmia scores, infarct size and cardiac mitochondrial dysfunction were prominently increased in NDO, HFO and HFS rats, compared with those in NDS rats. Treatment with either testosterone or vildagliptin similarly attenuated these impairments during I/R injury. These finding suggest that both testosterone replacement and vildagliptin share similar efficacy for cardioprotection during I/R injury by decreasing the infarct size and attenuating cardiac mitochondrial dysfunction caused by I/R injury in testosterone-deprived rats with obese insulin resistance.
\end{abstract}

\section{Key Words}

- obese testosterone deprivation

- dipeptidyl peptidase-4 inhibitors

- ischemia-reperfusion

- cardiac mitochondrial function
Journal of Endocrinology (2016) 231, 81-95
๑) 2016 Society for Endocrinology Printed in Great Britain
Published by Bioscientifica Ltd 


\section{Introduction}

Androgen deficiency occurs commonly in middle-aged to older men and also sometimes in younger men with underlying hypothalamopituitary or testicular disorders (Handelsman \& Zajac 2004). It is a primary risk factor for many disorders including obesity, metabolic syndrome, dyslipidemia, and especially coronary artery diseases (CAD) (Traish \& Kypreos 2011). Low testosterone levels have been shown to be related to CAD (Cornoldi et al. 2009) in which lower testosterone levels were associated with a higher prevalence of CAD (Wu \& von Eckardstein 2003).

Similar to increased aging population, the prevalence of obesity has increased dramatically over the past decades (Ogden et al. 2015). Long-term consumption of a high-fat diet (HFD) has been shown to cause not only obesity but also insulin resistance, a state in which the insulin receptors do not respond to insulin properly leading to impaired glycemic control (Pratchayasakul et al. 2011). The main causes of this impairment begins at an intracellular level, in which overproduction of reactive oxygen species (ROS) leads to increased oxidative stress and impaired insulin signaling cascade (Valle et al. 2011). This impairment can cause energy insufficiency in the heart, leading to serious progressive impairments including coronary artery disease (Espinola-Klein et al. 2011), acute myocardial infarction (Ducimetiere et al. 1980) and chronic heart failure (Krishnamoorthy et al. 2016). As a large number of aging men are found to be obese (Flegal et al. 2012), the adverse effect on the heart could be cumulative from both obese insulin resistance and testosterone deprivation.

In this study, the cardioprotective effects of testosterone and vildagliptin (dipeptidyl peptidase- 4 inhibitors) were investigated in testosterone-deprived and obese insulin-resistant model during ischemic and reperfusion (I/R) injury. The rational for the use of vildagliptin in orchiectomized rats in this study was due to the fact that previous studies demonstrated that testosterone deprivation is associated with the development of several disorders including obesity, insulin resistance and metabolic syndrome (Traish et al. 2009) as well as induced cardiovascular diseases and ischemic heart diseases (Kelly \& Jones 2013). Regarding the treatment, recent studies have demonstrated the beneficial effects of testosterone replacement and DPP4 inhibitor (vildagliptin) on insulin resistance and metabolic profile (Apaijai et al. 2014, Pintana et al. 2015) and on ischemic insults of the heart (Chinda et al. 2014, Pongkan et al. 2015). However, the effect of testosterone replacement and vildagliptin in the heart subjected with $\mathrm{I} / \mathrm{R}$ injury with testosterone deprivation combined with obese insulin resistance has never been investigated. Therefore, if vildagliptin exerts cardioprotection in testosteronedeprived subjects similar to testosterone replacement, it would be a great benefit and provide a new alternative treatment for the heart especially in elderly men who suffer from testosterone deprivation and metabolic syndrome, whereas testosterone replacement could not ameliorate peripheral insulin resistance (Garrido et al. 2014) and may cause undesirable effects (Reckelhoff et al. 2005). In this study, we tested the hypothesis that during cardiac I/R injury in obese insulin-resistant rats with testosterone deprivation, both testosterone and vildagliptin share similar efficacy in improving LV function and decreasing the incidence of arrhythmias and myocardial infarction size by attenuating cardiac mitochondrial dysfunction and apoptosis.

\section{Materials and methods}

\section{Animal preparation}

All experiments were approved by the Institutional Animal Care and Use Committee of the Faculty of Medicine, Chiang Mai University, Chiang Mai, Thailand. Male Wistar rats weighing $200 \mathrm{~g}$ were obtained from the National Laboratory Animal Center, Mahidol University, Bangkok, Thailand and were used for this study. Food and water were given ad libitum. The experimental protocol is illustrated in Fig. 1. For orchiectomy procedure, rats were anesthetized using isoflurane $2-3 \%$, and the orchiectomy was performed using the scrotal approach technique (Foley 2005). Rats vital signs were monitored throughout the operative time and observed for any other complications. Analgesic drugs and antibiotics were injected subcutaneously for 3 days after the operation.

\section{Surgical procedure of myocardial I/R}

Rats were anesthetized by intramuscular injection of Zoletil (zolazepam and tiletamine) $50 \mathrm{mg} / \mathrm{kg}$ combined with xylazine $3 \mathrm{mg} / \mathrm{kg}$, and the rats were ventilated with air via a tracheotomy by Harvard Rodent Ventilator Model 683 (Harvard Apparatus, Holliston, MA, USA) (Pongkan et al. 2015). Lead II electrocardiogram (ECG) (PowerLab $4 / 25 \mathrm{~T}$, AD Instrument) was recorded throughout the

Published by Bioscientifica Ltd. 


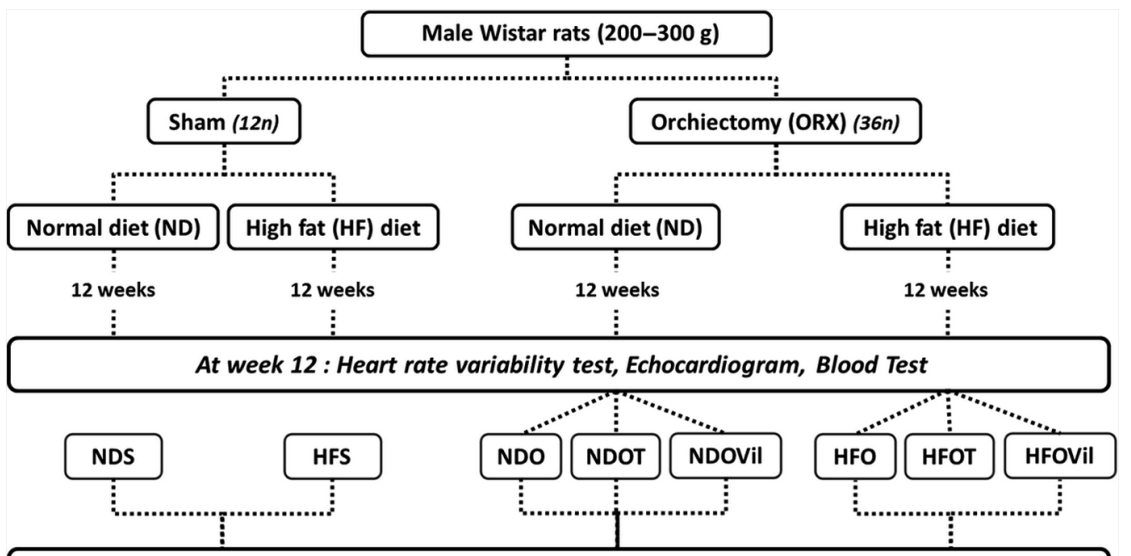

At week 16 : Heart rate variability test, Echocardiogram, Blood Test

I/R injury (LAD occlusion) $:$ Stabilize $=15 \mathrm{~min} /$ Ischemia $=\mathbf{3 0} \mathrm{min} /$ Reperfusion $=120 \mathrm{~min}$

Cardiac function and arrhythmia parameters were studied during $I / R$ injury. Then, the heart was removed to study the infarct size, mitochondrial function and western blot analysis.
Figure 1

Schematic representation of the study protocol. Orchiectomized or sham operated rats were divided to receive normal diet (ND) or high-fat diet (HFD) for 12 weeks. Then, orchiectomized rats in ND or HFD were divided to receive vehicle (castor oil; subcutaneously (s.c.)), testosterone $2 \mathrm{mg} / \mathrm{kg}$ BW (s.c. daily for 4 weeks) or vildagliptin (Galvas, Novartis) $3 \mathrm{mg} / \mathrm{kg}$ BW (intragastric gavage daily for 4 weeks), daily for 4 weeks. The cardiac function and HRV were determined at 12 th and 16 th week. I/R was performed by 30-min LAD ligation, followed by 120-min reperfusion. HFO, high fat + orchiectomy; HFOT, high fat+orchiectomy + testosterone; HFOVil, high fat + orchiectomy + testosterone; HFS, high fat+sham; NDO, normal diet + orchiectomy; NDOT, normal diet + orchiectomy + testosterone; NDOVil, normal diet + orchiectomy + vildagliptin; NDS, normal diet + sham; Sc., subcutaneous injection. study. The right carotid artery was cannulated with a pressure-volume (P-V) conductance catheter. The heart was exposed as described by previous study (Chinda et al. 2014). Ischemia was confirmed by an ST elevation on the ECG and regional pallor of myocardial tissues of the ischemic area. I/R injury was instigated by 30-min ischemia, followed by 120 -min reperfusion.

\section{Normal diet and high-fat diet preparation}

Standard laboratory chow (Mouse Feed Food No.082, C.P. Company, Bangkok, Thailand), considered as a normal diet (19.77\% of energy fat), was fed to the control rats. The high-fat diet (57.60\% of energy fat) consisted of the following ingredients: standard rat diet, casein, lard, cholesterol, vitamins, DL-methionine, yeast powder and sodium chloride (Pratchayasakul et al. 2011). All the ingredients were mixed and molded into a spherical shape. Rats were fed on these two diets for 12 weeks.

\section{Heart rate variability determination}

HRV is a noninvasive assessment of cardiac autonomic function, which is used to determine cardiac sympathovagal balance (Chattipakorn et al. 2007). In this experiment, HRV was analyzed at the 12th and 16th weeks of the study. An electrocardiogram (ECG) was conducted on each rat using PowerLab (PowerLab 4/25T, AD instrument, Bella Vista, NSW, Australia) with chart 5.0 continuously for $20 \mathrm{~min}$ while the animals were still conscious. Data from the ECG recording were analyzed using the MATLAB program and analyzed as described previously (Pongkan et al. 2015). An increased LF/HF ratio is indicative of cardiac sympathovagal imbalance (Chattipakorn et al. 2007).

\section{LV function determination}

LV function was determined using an echocardiograph (Vivid-i, GE) and was analyzed at the 12th and 16th weeks of the study. Rats were anesthetized and maintained with $2 \%$ isoflurane with oxygen $\left(2 \mathrm{LmL}^{-1}\right)$ inhalation. Signals from an M-mode echocardiogram at the level of the papillary muscles were recorded. Parameters obtained included right ventricular dimension during diastole, interventricular septum dimension during systole and diastole, LV internal dimension during systole (LVIDs) and diastole (LVIDd), and LV posterior wall thickness during systole and diastole. Fractional shortening (FS) was calculated using the formula: $\%$ FS $=($ LVIDd - LVIDs $) \times 100 /$ LVIDd (Rottman et al. 2007).

At the end of the experiment (week 16), LV function during I/R injury was evaluated using a P-V catheter (Scisense Instrument, Ontario, Canada). The right carotid artery was cannulated, and the pressurevolume relationship in the LV chamber was recorded using a LabScribe 2 program (iWorx Systems Inc, Dover, NH, USA) (Chinda et al. 2014). Heart rate (HR), LV end-systolic pressure (LVESP) and LV end-diastolic pressure (LVEDP), $P_{\max }, P_{\min }$, positive $d p / d t$, negative $\mathrm{dp} / \mathrm{dt}$ and stroke volume (SV) were assessed at the

Published by Bioscientifica Ltd. 
baseline, end of the ischemic period and at the end of the reperfusion period.

\section{Arrhythmia determination during I/R period}

ECG (Lead II) was monitored throughout the I/R period using a PowerLab 4/25 (AD Instruments, Inc, Colorado Springs, CO, USA). The occurrence of arrhythmia was characterized using Lambeth Conventions (Curtis et al. 2013), and arrhythmia scores were determined based on the following criteria as described previously (Pongkan et al. 2015).

\section{Infarct size determination}

At the end of the experiment, the heart was removed and the LAD was re-occluded. The heart was perfused by cold normal saline (Pongkan et al. 2015). Evans blue $(0.5 \%)$ was retrogradely infused through the aortic root. Nonstained area by Evans blue was defined as the area at risk (AAR). The heart was cut horizontally to 7-8 slices (Chinda et al. 2014), and then immersed and incubated in $37^{\circ} \mathrm{C}$ triphenyl tetrazolium chloride (TTC) for $12-15$ min to differentiate the infarct tissues (white area) from the viable myocardium (red area). The red and white areas were defined as the remote area, whereas only the white area was defined as the infarct area. The area measurements were calculated using the ImageTool software, version 3.0 (Chinda et al. 2014).

\section{Cardiac mitochondrial function study}

At the end of the experiment, cardiac mitochondria were isolated from nonischemic (remote area, R) and ischemic areas (I). The heart tissues were homogenized in a homogenizer as described previously (Pongkan et al. 2015). Cardiac mitochondrial functions were determined as mitochondrial reactive oxygen species (ROS) production, mitochondrial membrane potential change $(\Delta \Psi \mathrm{m})$ and mitochondrial swelling. Transmission electron microscopy (TEM) was also used to determine the morphology of the isolated cardiac mitochondria (Pongkan et al. 2015).

\section{Metabolic parameters, insulin resistance and testosterone level determination}

Fasting plasma glucose, total cholesterol and triglyceride concentrations were determined by an enzymatic colorimetric assay from a detection kit (Biotech, Bangkok, Thailand). Fasting plasma HDL was determined using colorimetric and fluorometric assays from a detection kit (BioVision), whereas plasma LDL was determined using Friedewald's equation using the fasting plasma HDL values (Friedewald et al. 1972). Fasting plasma insulin levels were determined using a sandwich ELISA (Millipore). Insulin resistance was assessed using the Homeostasis Model Assessment (HOMA) (Appleton et al. 2005). A higher HOMA index indicates a higher degree of insulin resistance (Pratchayasakul et al. 2011). Total plasma testosterone $(\mathrm{ng} / \mathrm{mL})$ was analyzed by ELISA at the Central Laboratory Service of Maharaj Nakorn Chiang Mai Hospital, Faculty of Medicine, Chiang Mai University.

\section{Western blot analysis}

Myocardial protein extract was prepared by homogenization of the frozen heart tissues in a lysis buffer (containing 1\% Nonidet P-40 (Calbiochem, Merck Millipore), $0.5 \%$ sodium deoxycholate, $0.1 \%$ sodium dodecyl sulfate (SDS) in $1 \times \mathrm{PBS}$ ). The total protein was mixed with a loading buffer and boiled at $95^{\circ} \mathrm{C}$ for $10 \mathrm{~min}$. Then, it was loaded into $10 \%$ gradient SDSpolyacrylamide gel and transferred to polyvinylidene difluoride (PVDF) membranes using transfer system (Bio-Rad). PVDF membranes were blocked with 5\% nonfat skimmed milk in TBST for $1 \mathrm{~h}$ (Pongkan et al. 2015). The membranes were exposed, overnight (at $4^{\circ} \mathrm{C}$ ), to anti- $\beta$-actin, anti-Bax (Santa Cruz Biotechnology), anti-Bcl-2, anti-caspase3, anti-total $\mathrm{Cx} 43$ and anti-Cx43 phosphorylated at S368 (Cell Signaling Technology) for $12 \mathrm{~h}$. On the next day, bound antibody was detected by horseradish peroxidase-conjugated with anti-mouse IgG. Enhanced chemiluminescence (ECL) detection reagents were used to visualize peroxidase reaction products. The band density was analyzed using ImageJ $1.47 \mathrm{~V}$ analysis (NIH image).

\section{Statistical analysis}

Data are expressed as mean \pm s.E.M. Biochemical parameters were compared using the Mann-Whitney $U$ test. VT/VF incidence was compared among groups using $\chi 2$ test. Statistical comparisons between groups were analyzed using Student's $t$-tests and one-way ANOVAs with post hoc LSD tests using SPSS, version 10.0. A $P$ value less than 0.05 was considered statistically significant.

Published by Bioscientifica Ltd 


\section{Results}

\section{Obesity, but not testosterone deficiency, caused peripheral insulin resistance, which was attenuated by vildagliptin and testosterone treatment}

Testosterone deprivation was confirmed by decreased plasma testosterone levels as well as the ratio of seminal vesicle to body weight (seminal vesicle ratio). Orchiectomized rats without/with obese insulin resistance in both dietary groups (NDO and HFO) had significantly decreased plasma testosterone levels and seminal vesicle ratios, when compared with the sham group (NDS and HFS). Testosterone replacement significantly increased plasma testosterone levels in orchiectomized rats (NDOT and HFOT) compared with those in the NDO and HFO groups, but was not different from the NDS group. However, the testosterone level in rats treated with vildagliptin (NDOVil and HFOVil) was still in the same level as that in the NDO and HFO groups (Table 1).

HFD rats showed a significant increase in body weight, compared with the ND group. Orchiectomized rats had significantly decreased body weight and visceral fat, compared with the sham rats in the same dietary groups. Interestingly, testosterone replacement significantly increased only the body weight in NDOT and HFOT rats, but not visceral fat, compared with that in NDO and
HFO groups, whereas vildagliptin treatment did not show any beneficial effects on body weight or visceral fat in NDOVil and HFOVil groups (Table 1). Moreover, peripheral insulin resistance developed only in the HFD groups (HFS and HFO), as shown by both increased plasma insulin levels and HOMA index, whereas total cholesterol levels and plasma LDL cholesterol levels were increased and plasma HDL cholesterol levels were decreased, compared with those of the NDS group (Table 1). No additive adverse effect in these parameters was found in the HFO group, compared with those in the HFS group, except in the case of plasma total cholesterol. Unlike rats in the HFD groups, NDO group did not have any significant alteration in these metabolic parameters, compared with the NDS group. These findings suggest that peripheral insulin resistance did not develop in the NDO rats.

Interestingly, testosterone replacement attenuated the severity of peripheral insulin resistance in the HFOT group, by decreasing plasma insulin levels and HOMA index, decreasing total cholesterol levels and decreasing plasma LDL cholesterol levels, whereas vildagliptin demonstrated the same beneficial effects in these parameters as well as increasing plasma HDL cholesterol levels in the HFOVil group, compared with the HFO group (Table 1). These findings suggest that testosterone replacement and vildagliptin treatment could attenuate

Table 1 Effects of testosterone and vildagliptin on metabolic parameters in testosterone-deprived rats at the end of week 16 ( $n=4-6 /$ group).

\begin{tabular}{|c|c|c|c|c|c|c|c|c|}
\hline \multirow[b]{2}{*}{ Parameters } & \multicolumn{4}{|c|}{ Normal diet } & \multicolumn{4}{|c|}{ High-fat diet } \\
\hline & NDS & NDO & NDOT & NDOVil & HFS & HFO & HFOT & HFOVil \\
\hline Body weight (g) & $517 \pm 11$ & $456 \pm 11^{a}$ & $494 \pm 2^{b}$ & $452 \pm 14^{a}$ & $605 \pm 16^{a}$ & $526 \pm 18^{b, c}$ & $572 \pm 17 \mathrm{a}, \mathrm{d}, \mathrm{e}$ & $522 \pm 17 \mathrm{c}, \mathrm{f}$ \\
\hline Visceral fat (g) & $36.3 \pm 3.4$ & $21.4 \pm 1.7^{a}$ & $24 \pm 1.8^{a}$ & $19.9 \pm 2.5^{\mathrm{a}}$ & $64.5 \pm 2.0^{a}$ & $41.6 \pm 1.5^{b, c}$ & $43.4 \pm 2.5^{\mathrm{a}, \mathrm{c}, \mathrm{e}}$ & $41.5 \pm 2.0^{c, f}$ \\
\hline $\begin{array}{l}\text { Plasma testosterone } \\
(\mathrm{ng} / \mathrm{mL})\end{array}$ & $2.9 \pm 0.5$ & $<0.025^{a}$ & $3.4 \pm 0.5^{b}$ & $<0.025^{a}$ & $0.5 \pm 0.1 \mathrm{a}$ & $<0.025^{c}$ & $3.9 \pm 0.7 c, d$ & $<0.025^{c}$ \\
\hline $\begin{array}{l}\text { Seminal vesicle } \\
\text { ratio }\left(\times 10^{3}\right)\end{array}$ & $4.10 \pm 0.02$ & $0.23 \pm 0.02^{a}$ & $4.06 \pm 0.1$ & $0.23 \pm 0.02^{a}$ & $2.75 \pm 0.14^{a}$ & $0.14 \pm 0.01^{a, c}$ & $4.20 \pm 0.4$ & $0.4 \pm 0.2^{a, c}$ \\
\hline $\begin{array}{l}\text { Plasma insulin } \\
(\mathrm{ng} / \mathrm{mL})\end{array}$ & $2.15 \pm 0.2$ & $2.2 \pm 0.3$ & $2.02 \pm 0.34$ & $1.93 \pm 0.36$ & $3.70 \pm 0.42^{\mathrm{a}}$ & $3.58 \pm 0.77^{a}$ & $1.94 \pm 0.40 \mathrm{c}, \mathrm{d}$ & $1.98 \pm 0.3 c, d$ \\
\hline $\begin{array}{l}\text { Plasma glucose } \\
(\mathrm{mg} / \mathrm{dL})\end{array}$ & $184.2 \pm 17.6$ & $173 \pm 7.1$ & $174 \pm 19.8$ & $179.4 \pm 8.7$ & $163.5 \pm 12.4$ & $184.7 \pm 8.8$ & $178.7 \pm 9.0$ & $179.6 \pm 11.3$ \\
\hline HOMA index & $18.7 \pm 2.5$ & $19.8 \pm 3.2$ & $19.1 \pm 3.5$ & $19.9 \pm 1.8$ & $32.25 \pm 3.7^{a}$ & $35.5 \pm 9.9^{a}$ & $16.1 \pm 2.1^{c, d}$ & $18.6 \pm 1.9 c, d$ \\
\hline $\begin{array}{l}\text { Plasma total } \\
\text { cholesterol (mg/ } \\
\text { dL) }\end{array}$ & $59.2 \pm 1.6$ & $62.7 \pm 2.3$ & $57.1 \pm 3.9$ & $59.3 \pm 4.1$ & $87.5 \pm 4.5^{a}$ & $101.2 \pm 4.8^{a, c}$ & $75.1 \pm 6.3^{a, d}$ & $80.8 \pm 6.6^{a, d}$ \\
\hline $\begin{array}{l}\text { Plasma triglyceride } \\
\text { (mg/dL) }\end{array}$ & $84.1 \pm 6.5$ & $83.3 \pm 14.0$ & $82.8 \pm 4.7$ & $80.1 \pm 5.7$ & $91.1 \pm 13.8$ & $81.6 \pm 6.2$ & $86.6 \pm 11.2$ & $83.2 \pm 5.2$ \\
\hline Plasma HDL (mg/dL) & $33.3 \pm 1.6$ & $36.4 \pm 1.4$ & $31.4 \pm 2.1$ & $34 \pm 1.3$ & $23.8 \pm 2.5^{a}$ & $25.7 \pm 2.0^{a}$ & $25.3 \pm 2.1^{a}$ & $33.7 \pm 2.4 c, d$ \\
\hline Plasma LDL (mg/dL) & $8.4 \pm 2.3$ & $9.5 \pm 2.7$ & $8.5 \pm 1.0$ & $10.1 \pm 3.0$ & $33.5 \pm 5.7 a$ & $54.4 \pm 5.6^{a}$ & $37.3 \pm 4.3^{a, d}$ & $37.7 \pm 4^{\mathrm{a}, \mathrm{d}}$ \\
\hline
\end{tabular}

HFO, high-fat diet + orchiectomy; HFOT, high-fat diet + orchiectomy + testosterone; HFOVil, high-fat diet + orchiectomy + vildagliptin; HFS, high-fat diet + sham surgery; HOMA, Homeostasis Model Assessment; MDA, malondialdehyde; NDO, normal diet + orchiectomy; NDOT, normal diet + orchiectomy + testosterone; NDOVil, normal diet +orchiectomy + vildagliptin; NDS, normal diet +sham surgery. a $P<0.05$ vs NDS, b $P<0.05$ vs NDO, $c P<0.05$ vs HFS, $\mathrm{d} P<0.05$ vs HFO, $\mathrm{e} P<0.05$ vs NDOT, $\mathrm{f} P<0.05$ vs NDOVil.

http://joe.endocrinology-journals.org DOI: $10.1530 / \mathrm{JOE}-16-0232$
(C) 2016 Society for Endocrinology Printed in Great Britain
Published by Bioscientifica Ltd 
peripheral insulin resistance in testosterone-deprived rats with obese insulin resistance by improving both insulin sensitivity and lipid profiles.

\section{Testosterone and vildagliptin preserved both} LV function and HRV in testosterone-deprived rats with obese insulin resistance before I/R injury

At week 12, LV dysfunction and sympathovagal imbalance were found in the NDO, HFS and HFO groups as indicated by decreased \%fractional shortening (Fig. 2A) and increased LF/HF ratio (Fig. 2C), compared with the NDS group. At week 16, impaired LV function and cardiac sympathovagal imbalance were found in NDO, HFS and HFO groups, and these parameters were not different between groups (Fig. 2B and D). Moreover, our results demonstrated that both testosterone replacement and vildagliptin treatment could preserve LV function and HRV by increased \%fractional shortening and decreased the LF/HF ratio in NDOT and NDOVil groups and HFOT and HFOVil groups, compared with that in NDO and HFO groups, respectively. No significant differences in
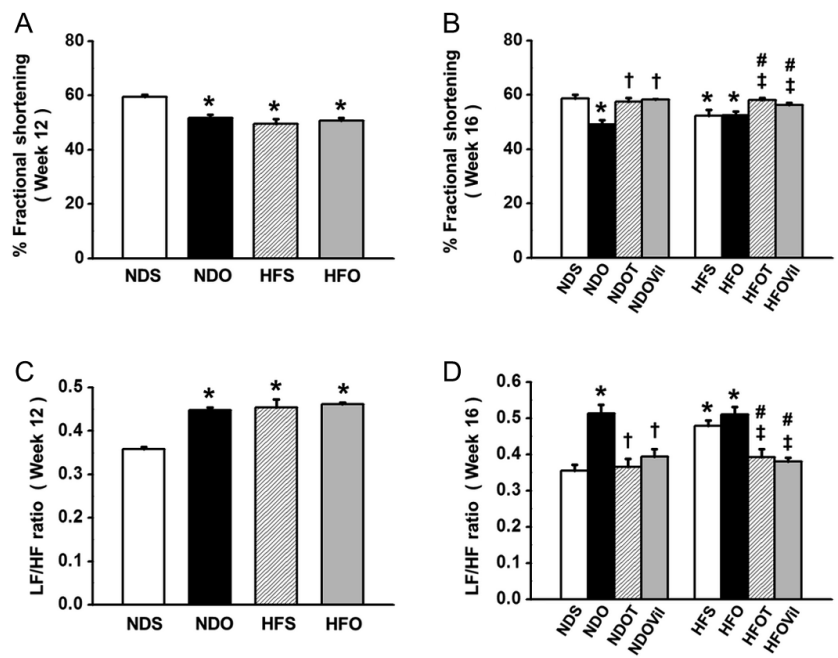

Figure 2

Effects of obese insulin resistance induced by long-term high-fat diet (HFD) consumption and testosterone deprivation at 12 weeks followed by testosterone and vildagliptin replacement on fractional shortening (FS) and heart rate variability (represented as low frequency/high frequency ratio: $\mathrm{LF} / \mathrm{HF}$ ratio) before $\mathrm{I} / \mathrm{R}$ injury. ( $\mathrm{A}$ and $\mathrm{B}$ ) Testosterone and vildagliptin restored the $\mathrm{FS}$ after treatment for 4 weeks, compared with the NDO and HFO groups. ( $C$ and D) Testosterone and vildagliptin reduced $\mathrm{LF} / \mathrm{HF}$ ratio after treatment for 4 weeks, compared with the NDO or HFO groups. ( ${ }^{*}<0.05$ vs NDS, ${ }^{+} P<0.05$ vs NDO, ${ }^{*} P<0.05$ vs HFS, $\# P<0.05$ vs HFO). HFO, high fat +orchiectomy; HFOT, high fat + orchiectomy + testosterone; HFOVil, high fat + orchiectomy + testosterone; HFS, high fat +sham; NDO, normal diet + orchiectomy; NDOT, normal diet + orchiectomy + testosterone; NDOVil, normal diet +orchiectomy + vildagliptin; NDS, normal diet+sham.

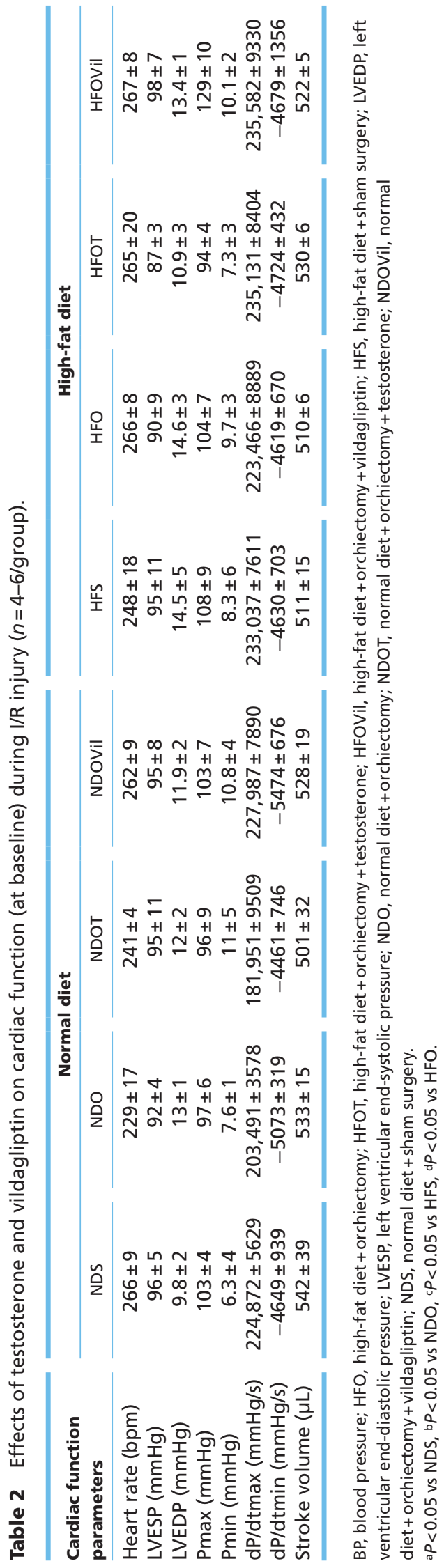

Published by Bioscientifica Ltd 


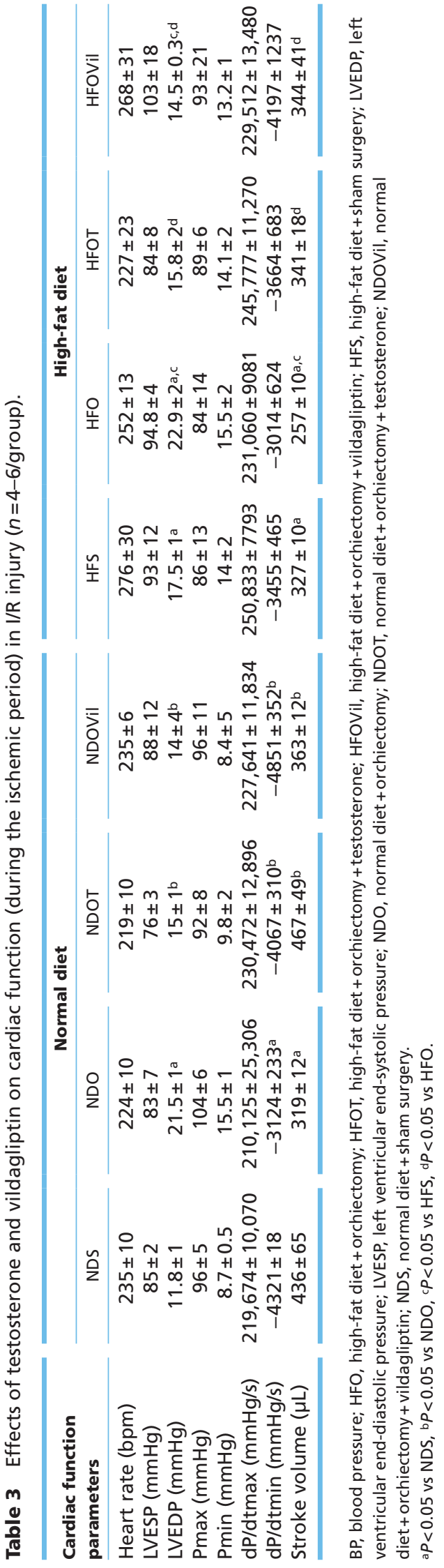

http://joe.endocrinology-journals.org DOI: $10.1530 / J O E-16-0232$
(๑) 2016 Society for Endocrinology Printed in Great Britain

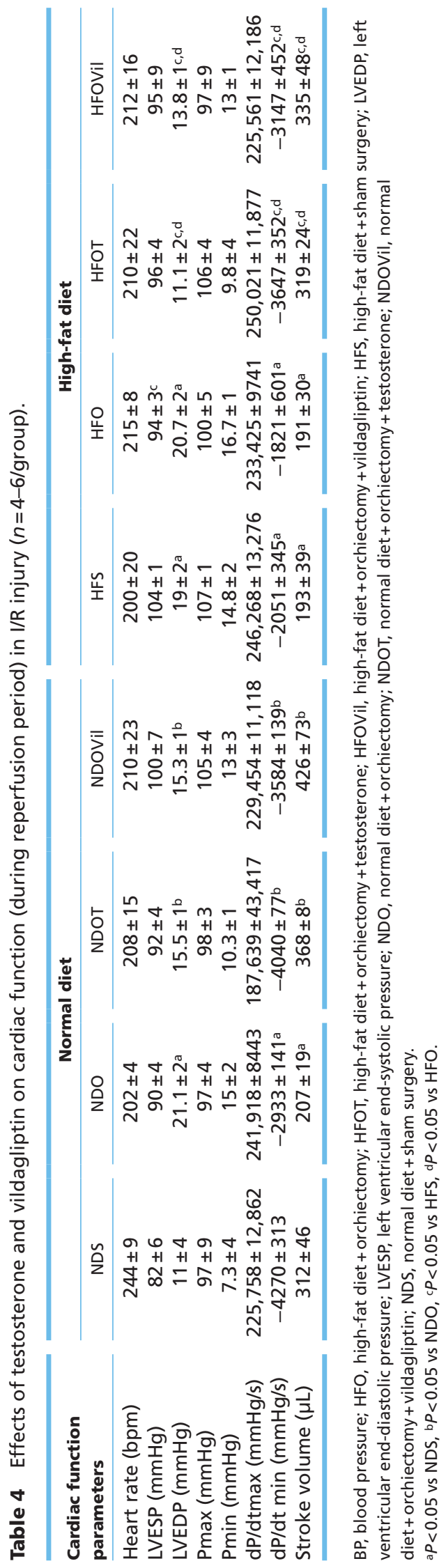

Published by Bioscientifica Ltd. 
\%fractional shortening and LF/HF ratio were found with testosterone and vildagliptin treatment in all groups (Fig. 2B and D). These findings suggest that testosterone replacement and vildagliptin treatment could preserve LV function and HRV by increasing \%fractional shortening and decreasing the LF/HF ratio in testosterone-deprived rats with obese insulin resistance.

\section{Testosterone and vildagliptin preserved LV function during I/R injury in testosterone-deprived rats with obese insulin resistance}

The changes in LV function parameters during I/R injury are shown in Tables 2,3 and 4. At the baseline, $\mathrm{LV}$ functions were not different between any groups (Table 2). During $\mathrm{I} / \mathrm{R}$ injury (at the end of ischemia), the NDO group had increased LVEDP and $\mathrm{dP} / \mathrm{dtmin}$ as well as decreased stroke volume, compared with the NDS group. Interestingly, testosterone and vildagliptin treatment in the ND group significantly decreased LVEDP, dP/dtmin and increased stroke volume, compared with the NDO group. In addition, the HFD group (HFS and HFO group) had increased LVEDP and deceased stroke volume compared with the NDS group. However, the HFO group demonstrated greater severity in these parameters than the HFS group. Both testosterone and vildagliptin treatment in the HFD group could attenuate LV dysfunction by significantly decreasing LVEDP and increasing stroke volume, compared with that in the HFO group (Table 3). At the end of reperfusion, most parameters remained similar to those during the ischemic period except that rats in the HFO group had decreased LVESP, compared with those in the HFS group, suggesting that testosterone and vildagliptin treatment in the HFD group could attenuate LV dysfunction by significantly decreasing LVEDP, dp/dtmin and increasing stroke volume, when compared with the HFO group (Table 4). These findings suggest that testosterone replacement and vildagliptin treatment could attenuate LV dysfunction by decreasing diastolic dysfunction and increasing stroke volume in testosterone-deprived rats with obese insulin resistance.

\section{Testosterone replacement and vildagliptin decreased the occurrence of cardiac arrhythmias in testosterone- deprived rats with obese insulin resistance during I/R injury}

The VT/VF incidence during I/R injury was not different between groups (Fig. 3A). However, in the NDO group,

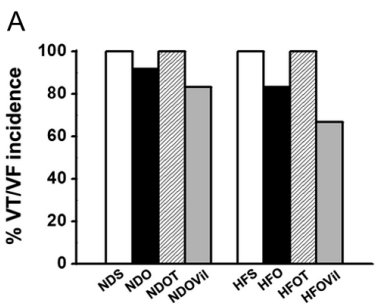

$\mathrm{B}$
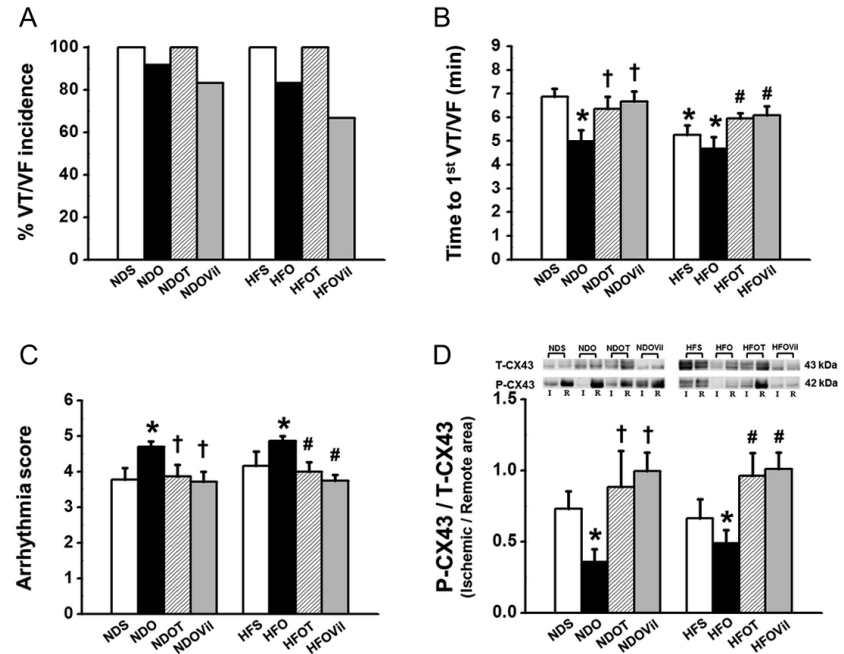

Figure 3

Effects of testosterone and vildagliptin on the occurrence of cardiac arrhythmia during the ischemic-reperfusion period in a normal diet-fed, obese insulin-resistant with testosterone deprivation model. Testosterone and vildagliptin did not reduce: (A) VT/VF incidence in normal diet and obese insulin-resistant and testosterone-deprived rats but reduced cardiac arrhythmia by increasing (B) time to 1st VT/NF incidence and decreased (C) arrhythmia score, when compared with the NDO or HFO groups. (D) Testosterone and vildagliptin increased $\mathrm{P}-\mathrm{C} \times 43 / \mathrm{T}-\mathrm{C} \times 43$ ratio when compared with the NDO and HFO groups. ( ${ }^{*} P<0.05$ vs NDS, $+P<0.05$ vs NDO, $\# P<0.05$ vs HFO). HFO, high fat + orchiectomy; HFOT, high fat+orchiectomy + testosterone; HFOVil, high fat + orchiectomy + testosterone; HFS, high fat +sham; I, ischemic area; NDO, normal diet +orchiectomy; NDOT, normal

diet + orchiectomy + testosterone; NDOVil, normal diet + orchiectomy + vildagliptin; NDS, normal diet +sham; R, remote area; VF, ventricular fibrillation; VT, ventricular tachycardia.

the time to first VT/VF onset was significantly decreased, whereas the arrhythmia score was significantly greater, compared with the NDS group. Interestingly, testosterone replacement and vildagliptin treatment significantly reduced cardiac arrhythmia by increasing the time to 1st VT/VF (Fig. 3B) and reducing the arrhythmia score during I/R injury (Fig. 3C), when compared with the NDO group. The HFS group demonstrated a decreased time to 1st VT/VF and showed no difference in arrhythmia score, compared with the NDS group, whereas the HFO group demonstrated a decreased time to the 1st VT/VF and increased arrhythmia during I/R injury, compared with the NDS group. However, time to 1st VT/VF and arrhythmia score in the HFS and HFO group showed no difference. Interestingly, testosterone replacement and vildagliptin could increase the time to 1st VT/VF (Fig. 3B) and reduce arrhythmia score during I/R injury (Fig. 3C), compared with the HFO group.

Our study demonstrated that the phosphorylated $\mathrm{Cx} 43$ per total $\mathrm{Cx} 43$ ratio (P-Cx43/T-Cx43 ratio) of the ischemic-to-remote areas was significantly decreased

Published by Bioscientifica Ltd. 
in NDO and HFO groups, whereas all treatment groups (NDOT, HFOT, NDOVil and HFOVil) had a significantly increased P-Cx43/T-Cx43 ratio, compared with sham rats being fed with the same diets (Fig. 3D). These findings suggest that both testosterone replacement and vildagliptin treatment can attenuate cardiac arrhythmia during I/R injury in testosterone-deprived rats with obese insulin resistance.

\section{Testosterone replacement and vildagliptin treatment reduced the infarct size in the I/R heart of testosterone- deprived rats with obese insulin resistance}

The area at risk (AAR) was not different between all groups. However, the infarct size of NDO, HFO and HFS groups were significantly greater compared with the NDS group. Interestingly, testosterone and vildagliptin reduced the infarct size by 35.7 and $44.1 \%$, respectively, when compared with that in the NDO group and also a reduced infarct size by 55.4 and $49.5 \%$, respectively, compared with that in the HFO group (Fig. 4A). Representative images of the infarct size are shown in Fig. 4B.

Western blot analysis demonstrated that the expression of pro-apoptotic/anti-apoptotic protein (Bax/Bcl-2 ratio) was markedly increased in NDO and HFO groups (Fig. 4C), whereas the expression of pro-caspase 3 was not significantly different in the NDO and HFO groups (Fig. 4D), when compared with the NDS group. Interestingly, testosterone replacement and vildagliptin in $\mathrm{ND}$ and $\mathrm{HFD}$ decreased $\mathrm{Bax} / \mathrm{Bcl}-2$ ratio and increased pro-caspase 3 expression, compared with the NDO and HFO groups, respectively (Fig. 4C and D). These findings suggest that testosterone replacement and vildagliptin treatment effectively reduced the infarct size by attenuating apoptotic processes in testosteronedeprived rats with obese insulin resistance.

\section{Testosterone replacement and vildagliptin treatment attenuated mitochondrial dysfunction in the I/R heart of testosterone-deprived rats with obese insulin resistance}

There were no differences among groups in ROS production levels, mitochondrial depolarization or mitochondrial swelling of cardiac mitochondria taken from the nonischemic myocardium. Cardiac mitochondria taken from the ischemic areas of the NDO, HFS and HFO groups had significantly increased mitochondrial ROS (Fig. 5A), increased mitochondrial depolarization indicated by a decrease in the red/green fluorescent intensity ratio (Fig. 5B), and increased mitochondrial swelling indicated by a decrease in absorbance at $540 \mathrm{~nm}$ (Fig. 5C), when compared with that from the NDS group.

Testosterone replacement and vildagliptin treatment in both ND and HFD groups attenuated mitochondrial dysfunction in the ischemic myocardium of all ORX groups as indicated by reduced ROS production (Fig. 5A), attenuated mitochondrial depolarization (Fig. 5B) and
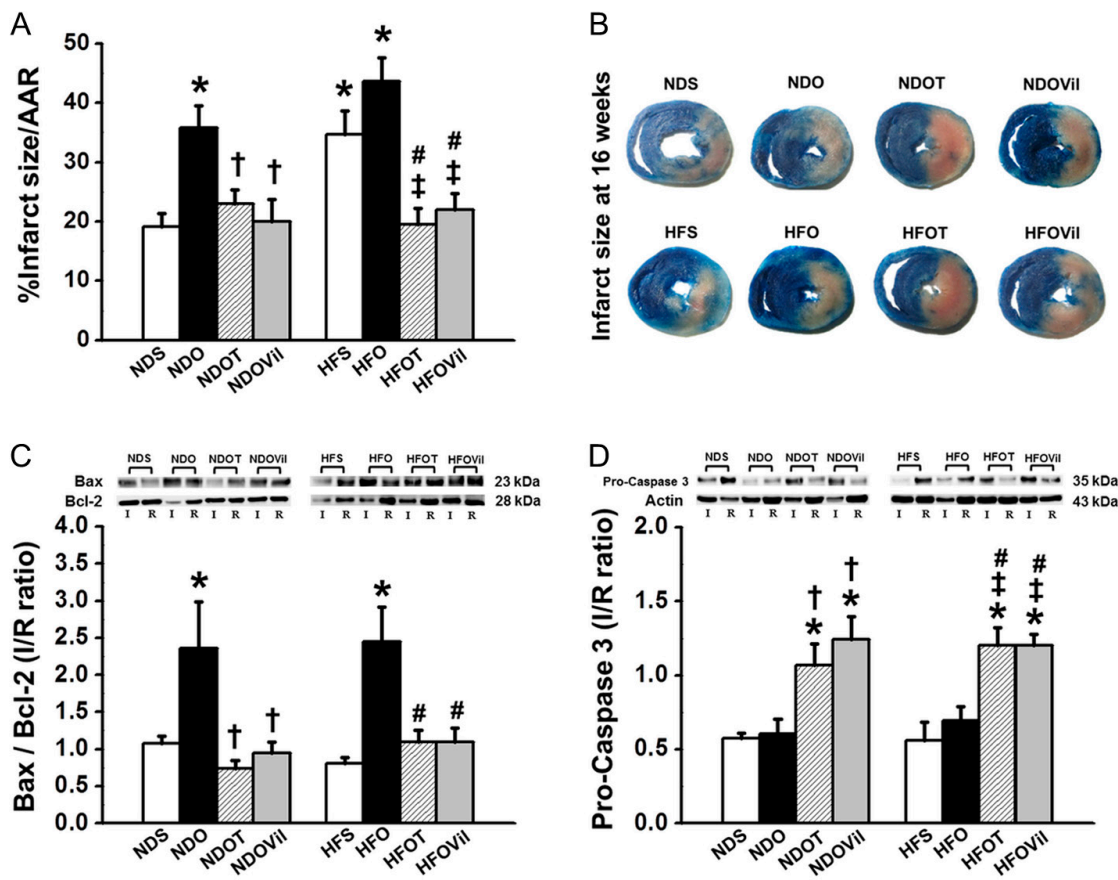

Figure 4

Effects of testosterone and vildagliptin on the infarct size, anti-apoptotic and pro-apoptotic proteins after ischemic-reperfusion period in a normal diet-fed, obese-insulin resistant with testosterone deprivation model. The area at risk (AAR) was not different between all groups. ( $A$ and $B$ ) Testosterone and vildagliptin reduced the infarct size by 35.7 and $44.1 \%$, respectively, compared with those in the NDO group and reduced the infarct size by 55.4 and $49.5 \%$, respectively, compared with those in the HFO group. Testosterone and vildagliptin attenuated apoptotic process (C) by decreasing proapoptotic/anti-apoptotic ratio (Bax/BCl-2 ratio) and (D) increasing pro-caspase3 expression, compared with the NDO and HFO groups ( ${ }^{*} P<0.05$ vs NDS, ${ }^{+} P<0.05$ vs NDO, ${ }^{\ddagger} P<0.05$ vs HFS, $\# P<0.05$ vs HFO). HFO, high fat + orchiectomy; HFOT, high fat + orchiectomy+ testosterone; HFOVil, high fat + orchiectomy + testosterone; HFS, high fat + sham; I, ischemic area; NDO, normal diet+orchiectomy; NDOT, normal diet +orchiectomy + testosterone; NDOVil, normal diet + orchiectomy+ vildagliptin; NDS, normal diet + sham; R, remote area. http://joe.endocrinology-journals.org DOI: $10.1530 / J O E-16-0232$
() 2016 Society for Endocrinology Printed in Great Britain 
A

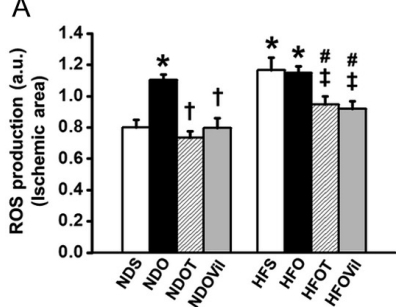

B
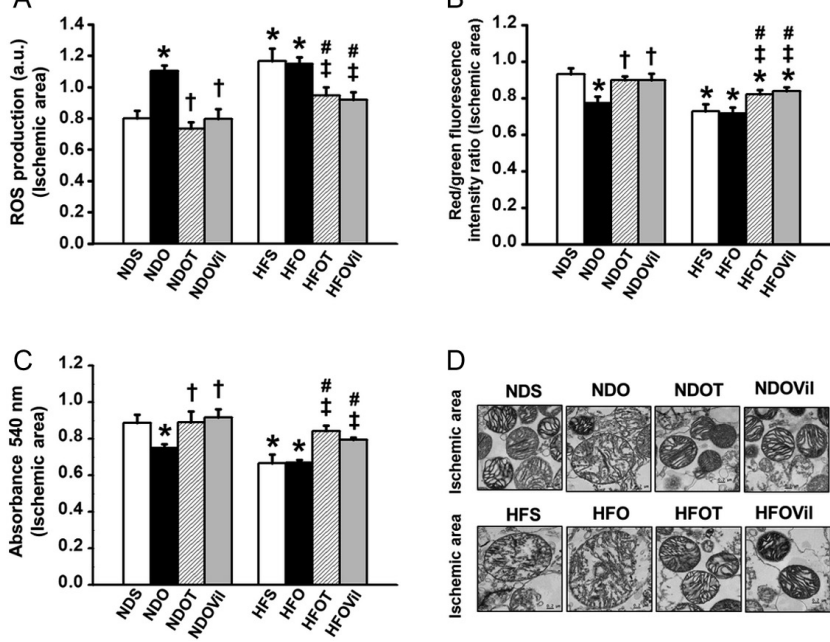

Figure 5

Effects of testosterone and vildagliptin on cardiac mitochondria after ischemic-reperfusion period in a normal diet-fed, obese insulin-resistant with testosterone deprivation model. Testosterone and vildagliptin improved cardiac mitochondrial function by (A) reducing reactive oxygen species (ROS) production, (B) attenuating mitochondrial membrane depolarization and (C) attenuating mitochondrial swelling in ischemic areas. (D) Testosterone and vildagliptin also attenuated the deterioration of cardiac mitochondrial morphology ( $* P<0.05$ vs NDS, ${ }^{+} P<0.05$ vs NDO, ${ }^{\ddagger} P<0.05$ vs HFS, $\sharp P<0.05$ vs HFO). HFO, high fat + orchiectomy; HFOT, high fat + orchiectomy + testosterone; HFOVil, high

fat + orchiectomy + testosterone; HFS, high fat +sham; NDO, normal diet + orchiectomy; NDOT, normal diet + orchiectomy + testosterone; NDOVil, normal diet + orchiectomy + vildagliptin; NDS, normal diet + sham; ROS, reactive oxygen species.

reduced mitochondrial swelling (Fig. 5C), compared with the ORX group of the same diet regimen. Representative transmission electron microscope (TEM) pictures of cardiac mitochondria in the ischemic myocardium demonstrated that NDO, HFO and HFS groups had more severe mitochondrial swelling, which was indicated by abnormal morphology together with the loss of cristae in the cardiac mitochondrion, than the NDS and ORX group on testosterone or vildagliptin treatment (Fig. 5D). These findings suggest that testosterone replacement and vildagliptin treatment shared similar efficacy in attenuating the cardiac mitochondrial dysfunction by reducing cardiac mitochondrial ROS production, mitochondrial depolarization and mitochondrial swelling in the I/R hearts of testosterone-deprived rats with obese insulin resistance.

\section{Discussion and conclusions}

This study has demonstrated the potential cardioprotective effects of testosterone replacement and DPP-4 inhibitor on the $I / R$ hearts of testosterone-deprived rats with obese insulin resistance. The major findings of this study are as follows. First, high-fat diet, but not testosterone deprivation, induced obese insulin resistance, whereas testosterone replacement and DPP-4 inhibitor (vildagliptin) treatment improved insulin sensitivity and metabolic parameters in testosterone-deprived rats with obese insulin resistance. Secondly, LV function and the HRV were impaired with testosterone deprivation in both obese and nonobese insulin resistance models. Thirdly, testosterone and vildagliptin provided cardioprotective effects in cases of testosterone deprivation alone and in a testosterone-deprived rat with obese insulin resistance model. Fourthly, during the I/R period, testosterone and vildagliptin exerted cardioprotective effects in a testosterone deprivation model and in a testosterone deprivation with obese insulin resistance model, identified by improving LV pressure and stroke volume, reducing cardiac arrhythmias, decreasing the infarct size and attenuating cardiac mitochondrial dysfunction caused by I/R injury.

This study demonstrated that testosterone deprivation alone (NDO group) neither induced insulin resistance nor had adverse effects on metabolic parameters. On the other hand, HFS and HFO groups developed insulin resistance, which is consistent with previous studies which reported that long-term consumption of a HFD caused obese insulin resistance, which is indicated both by increased plasma insulin levels and HOMA index (Pratchayasakul et al. 2011, Pintana et al. 2015, Pongkan et al. 2016). As the severity of insulin resistance at 16 weeks was not significantly different between the HFS and HFO group, this finding suggests that these two conditions were not additive in terms of insulin resistance. Therefore, this finding suggests that these two conditions were not additive in terms of insulin resistance. In this study, our results further demonstrated that testosterone deprivation also did not aggravate the severity of the cardiac impairments such as LV dysfunction and cardiac sympathovagal imbalance in the obese insulin resistant model.

In addition, low testosterone levels and obese insulin resistance are known as risk factors of cardiac dysfunction (Culic \& Busic 2015) due mainly to increased oxidative stress (Hwang et al. 2011) in the heart, and leading to depressed HRV (Brook et al. 2010). Moreover, previous studies found the association of low testosterone and the increase in the reactive oxygen species (ROS) level (Pongkan et al. 2015, 2016). Lateef and coworkers demonstrated that low testosterone increased the

Published by Bioscientifica Ltd. 
synthesis of nitric oxide (NO), which could upregulate urokinase plasminogen activation receptor (UPAR) expression, resulting in plasmin formation, which activates matrix metalloproteinase 9 (MMP-9), leading to extracellular matrix degradation resulting in increased ROS, especially in the mitochondria (Lateef et al. 2013). Moreover, they found that ROS production from extracellular matrix disruption also induced lipid peroxidation and caused the reduction of antioxidant levels such as glutathione reductase, catalase and glutathione-dependent enzyme (Lateef et al. 2013). When the accumulation of ROS in mitochondria reach a critical threshold level, it triggers the opening of the mitochondrial permeability transition pores (MPTP), resulting in induced mitochondrial membrane depolarization (Hausenloy et al. 2003). Moreover, the prolonged opening of MPTP could lead to an increase in the matrix osmotic pressure, resulting in mitochondrial swelling and/or mitochondrial membrane rupture. This will cause the release of proapoptotic cytochrome $\mathrm{C}$ and other pro-apoptotic proteins, leading to apoptotic cell death (Halestrap \& Pasdois 2009). The increase in apoptotic cells induces the enlargement of myocardial infarct size and, as myocardial infarction plays an essential role in cardiac dysfunction, reduction in the infarct size would be of great benefit regarding contractility. This study is consistent with previous studies, demonstrating that NDO, HFS and HFO groups exhibit cardiac dysfunction and depressed HRV in both 12 and 16 weeks.

Regarding therapeutic outcomes, after treatment with a physiological dose of testosterone for 4 weeks, the level of plasma testosterone in the NDOT and HFOT groups was restored back to the same level as the NDS group, whereas plasma testosterone levels in the HFS was significantly lower than those in the NDS and testosterone-treated groups. In NDOVil and HFOVil groups, the level of testosterone was not altered. Interestingly, our study demonstrated that testosterone replacement and vildagliptin treatment in ORX rats with obese insulin resistance (HFO) improved insulin resistance by decreasing plasma insulin levels, HOMA index, total cholesterol levels and plasma LDL cholesterol levels. This is consistent with our previous studies (Apaijai et al. 2012, Pintana et al. 2015) as well as others (Traish et al. 2014, Bayram et al. 2016), which demonstrated that testosterone replacement and vildagliptin treatment could attenuate insulin resistance with testosterone deprivation with obese insulin resistance model. These findings suggest that vildagliptin gives beneficial effects similar to testosterone replacement by improving insulin resistance and other metabolic parameters in testosterone-deprived rats with obese insulin resistance. Moreover, decreasing insulin resistance in several anti-diabetic drugs of different classes such as metformin, liraglutide and vildagliptin demonstrated the cardioprotective effect (Apaijai et al. 2012, 2014, Barreto-Vianna et al. 2016). However, DPP-4 inhibitor itself might exert direct beneficial effects on the cardiomyocyte, for example, promote the endothelial cell function and upregulate protein expression resulting in cardioprotection (Giannocco et al. 2013, Ishii et al. 2014).

In addition to the benefits on metabolic parameters, testosterone replacement and vildagliptin treatment also exhibited similar beneficial effects on cardiac function and HRV in ORX rats with/without obese insulin resistance (HFO and NDO groups) by increasing \%fractional shortening and decreasing the LF/HF ratios, respectively, when compared with the ORX group. Although previous studies demonstrated that either testosterone or vildagliptin exerted beneficial effects on cardiac function in HFO and NDO groups in a testosterone-deprived model (Pongkan et al. 2015), obese-insulin resistance model (Apaijai et al. 2012, 2014) and testosterone-deprived with obese-insulin resistance model (Francomano et al. 2014), our study is the first to compare the efficacy of testosterone replacement and vildagliptin for their cardioprotection. Regarding the effects of testosterone replacement and vildagliptin on the suppression of mitochondrial ROS production and apoptosis, previous studies demonstrated that either testosterone (Hwang et al. 2011, Zhang et al. 2013, Pintana et al. 2015) or vildagliptin (Avila Dde et al. 2013, Apaijai et al. 2014, Chinda et al. 2014) could effectively reduce ROS generation and suppressed oxidative stress. In addition, Alessandro and coworkers reported that testosterone decreased ROS production by binding to cytoplasmic androgen receptor (AR) and promoting its translocation to the nucleus, resulting in enhanced extracellular matrix (ECM) production via the transcription-inducing complex c-FOS/c-JUN (Bertolo et al. 2014). Moreover, previous studies demonstrated that both testosterone (Mancini et al. 2008, Zhang et al. 2013) and vildagliptin (Avila Dde et al. 2013, El-Sherbeeny \& Nader 2016) could decrease oxidative stress by increasing the antioxidative substance such as catalase (CAT), superoxide dismutase (SOD) and glutathione peroxidase (GPx). This hypothesis is supported by the findings in this study that HFO and NDO groups had increased cardiac mitochondrial ROS production and that both testosterone and vildagliptin effectively attenuated cardiac mitochondrial ROS levels and reduced mitochondrial dysfunction (Chinda et al. 2014, Pongkan et al. 2015). Therefore, it is possible

Published by Bioscientifica Ltd 
that the enhancement of the antioxidant defense system and reduction in the levels of oxidative stress of testosterone and vildagliptin could be responsible for their cardioprotection. As both testosterone replacement and vildagliptin share similar efficacy in cardioprotection, these findings could provide an implication for future clinical treatment options in those patients who cannot be treated with testosterone.

During the I/R injury, the results clearly demonstrated that the HFS, HFO and NDO groups demonstrated an impairment of LV function, whereas either testosterone or vildagliptin exerted similar efficacy in cardioprotection in this case. This could be due to their similar efficacy in reducing the infarct size and improving cardiac mitochondrial function, which was impaired during I/R injury. Moreover, as myocardial infarct size has been shown to be associated with the severity of left ventricular dysfunction (Chinda et al. 2014), reduction in the infarct size would be of great benefit regarding improved LV function. In this study, HFS, HFO and NDO groups demonstrated a larger infarct size/area at risk than NDS group. Interestingly, either testosterone or vildagliptin exerted similar efficacy in reducing the infarct size in this case. Previous studies on myocardial apoptosis attenuation demonstrated that testosterone replacement could reduce the myocardial infarct size and the potential mechanism could be due to reduced apoptosis and the reduction of cardiac mitochondrial dysfunction, which was demonstrated that testosterone attenuated myocardial apoptosis by increasing anti-apoptotic (Bcl-2) proteins and reducing pro-apoptotic (Bax) proteins (Pongkan et al. 2015). Moreover, Huang and coworkers demonstrated that chronic exogenous testosterone replacement improved cardiac function via Akt/PKB signaling pathway resulting in decreased pro-apoptotic (Bax, Fas-L protein) and increased anti-apoptotic proteins (Bcl-2, p-Bad, p-FOXO3a) in isolated heart investigation (Huang et al. 2010). For vildagliptin, it has been shown that this drug reduced the infarct size by attenuating myocardial apoptosis via increased anti-apoptotic (Bcl-2) proteins (Apaijai et al. 2014, Chinda et al. 2014), increased pro-caspase 3 (Chinda et al. 2014) and decreased pro-apoptotic (Bax) proteins (Apaijai et al. 2014) in $\mathrm{I} / \mathrm{R}$ injury model as well as renal ischemia-reperfusion injury model (Glorie et al. 2012). Moreover, treatment with DPP4 inhibitor in obese, prediabetic (DIO) rats for 4 weeks has been shown to reduce myocardial infarct size after $\mathrm{I} / \mathrm{R}$ via an increase of the phosphorylation of Akt/PKB signaling proteins, which involved in the RISK (reperfusion-induced salvage kinase) pathway
(Huisamen et al. 2011). Consistent with previous studies, our results demonstrated that testosteroneand vildagliptin attenuated cardiac mitochondrial dysfunction, which is indicated by decreased mitochondrial ROS production, mitochondrial depolarization and mitochondrial swelling as well as attenuated myocardial apoptosis by decreased Bax/Bcl-2 ratio and increased pro-caspase 3 expression. This study also showed that I/R injury induced apoptosis by decreasing pro-caspase3, which is consistent with previous reports in models of $\mathrm{I} / \mathrm{R}$ injury and obesity (Lu et al. 2007, 2016). Increased pro-caspase3 has been shown to play a beneficial role to the cell and acts as the critical point of apoptotic process because pro-caspase 3 is an inactive form until it is converted to cleaved caspase 3, which is an active form that induced apoptosis (Earnshaw et al. 1999, Peterson et al. 2009, Walters et al. 2009). Mitochondria are the organelles, which play an essential role in cell survival as well as regulation of cell death, especially in cardiomyocytes and increasing intracellular oxidative stress during I/R injury could lead to cardiomyocyte swelling and death (Pongkan et al. 2015). In this study, we are reporting for the first time similar beneficial effects of testosterone replacement and vildagliptin during I/R injury in an HFO model, and our findings demonstrate that vildagliptin exhibited similar beneficial effects on the infarct size reduction and improved cardiac mitochondrial function during I/R injury as did testosterone replacement in the HFO and NDO models.

Regarding cardiac arrhythmias, as cardiac mitochondrial depolarization and decreased phosphorylation of $\mathrm{Cx} 43$ have been shown to facilitate cardiac arrhythmias (Aon et al. 2009, Chinda et al. 2014), interventions that prevent these two events could attenuate the arrhythmia incidence (Maruyama et al. 2016). Our previous studies (Apaijai et al. 2014, Pongkan et al. 2015) also demonstrated that both testosterone and vildagliptin could attenuate mitochondrial depolarization and increased phosphorylation of $\mathrm{Cx} 43$. Our results in this study demonstrated that the ORX groups were more susceptible to arrhythmias as indicated by decreased time to 1st VT/VF onset and increased arrhythmia scores compared with the sham group of its own diet assignment. As treated groups (NDOT, NDOVil, HFOT and HFOVil) had longer times to 1st VT/VF onset and lower arrhythmia scores, compared with the ORX group, these findings indicated that testosterone and vildagliptin exerted similar antiarrhythmic effects in NDO and HFO groups during I/R injury.

Published by Bioscientifica Ltd 
In conclusion, before $\mathrm{I} / \mathrm{R}$ injury, vildagliptin treatment exhibits similar cardioprotective efficacy as testosterone replacement in testosterone-deprived rats with obese insulin resistance caused by longterm consumption of a HFD, by improving cardiac sympathovagal balance and LV function. During I/R injury, vildagliptin treatment and testosterone replacement also exerted similar cardioprotective effects by ameliorating left ventricular dysfunction and reducing the infarct size and cardiac arrhythmias caused by I/R injury under testosterone deprivation with obese insulin-resistant conditions. These beneficial effects of both testosterone and vildagliptin were due to their ability to increase $\mathrm{Cx} 43$ phosphorylation and attenuate cardiac mitochondrial dysfunction and cardiomyocyte apoptosis. These findings suggest that vildagliptin may be used as an alternative to testosterone in testosteronedeprived subjects with or without obese insulin resistance. Whether the combined treatment of vildagliptin and testosterone replacement would provide the synergistic effects needs to be investigated in the future.

\section{Declaration of interest}

The authors declare that there is no conflict of interest that could be perceived as prejudicing the impartiality of the research reported.

\section{Funding}

This work was supported by the NSTDA Research Chair Grant from the National Science and Technology Development Agency Thailand (N C) and grants from the Thailand Research Fund BRG5780016 (S C), the Royal Golden Jubilee PhD Program (N C and W P), the National Research Council Thailand (N C) and the Chiang Mai University Center of Excellence Center Award (N C).

\section{References}

Aon MA, Cortassa S, Akar FG, Brown DA, Zhou L \& O’Rourke B 2009 From mitochondrial dynamics to arrhythmias. International Journal of Biochemistry and Cell Biology 41 1940-1948. (doi:10.1016/ j.biocel.2009.02.016)

Apaijai N, Pintana H, Chattipakorn SC \& Chattipakorn N 2012 Cardioprotective effects of metformin and vildagliptin in adult rats with insulin resistance induced by a high-fat diet. Endocrinology 153 3878-3885. (doi:10.1210/en.2012-1262)

Apaijai N, Chinda K, Palee S, Chattipakorn S \& Chattipakorn N 2014 Combined vildagliptin and metformin exert better cardioprotection than monotherapy against ischemia-reperfusion injury in obeseinsulin resistant rats. PLOS ONE 9 e102374. (doi:10.1371/journal. pone.0102374)

Appleton DJ, Rand JS \& Sunvold GD 2005 Basal plasma insulin and homeostasis model assessment (HOMA) are indicators of insulin sensitivity in cats. Journal of Feline Medicine and Surgery 7 183-193. (doi:10.1016/j.jfms.2004.12.002)

Avila Dde L, Araujo GR, Silva M, Miranda PH, Diniz MF, Pedrosa ML, Silva ME, de Lima WG \& Costa DC 2013 Vildagliptin ameliorates oxidative stress and pancreatic beta cell destruction in type 1 diabetic rats. Archives of Medical Research 44 194-202. (doi:10.1016/ j.arcmed.2013.03.004)

Barreto-Vianna AR, Aguila MB \& Mandarim-de-Lacerda CA 2016 Effects of liraglutide in hypothalamic arcuate nucleus of obese mice. Obesity 24 626-633. (doi:10.1002/oby.21387)

Bayram F, Elbuken G, Korkmaz C, Aydogdu A, Karaca Z \& Cakir I 2016 The effects of gonadotropin replacement therapy on metabolic parameters and body composition in men with idiopathic hypogonadotropic hypogonadism. Hormone and Metabolic Research 48 112-117. (doi:10.1055/s-0035-1564252)

Bertolo A, Baur M, Aebli N, Ferguson SJ \& Stoyanov J 2014 Physiological testosterone levels enhance chondrogenic extracellular matrix synthesis by male intervertebral disc cells in vitro, but not by mesenchymal stem cells. Spine Journal 14 455-468. (doi:10.1016/ j.spinee.2013.10.018)

Brook RD, Rajagopalan S, Pope CA 3rd, Brook JR, Bhatnagar A, Diez-Roux AV, Holguin F, Hong Y, Luepker RV, Mittleman MA et al. 2010 Particulate matter air pollution and cardiovascular disease: an update to the scientific statement from the American Heart Association. Circulation 121 2331-2378. (doi:10.1161/ CIR.0b013e3181dbece1)

Chattipakorn N, Incharoen T, Kanlop N \& Chattipakorn S 2007 Heart rate variability in myocardial infarction and heart failure. International Journal of Cardiology 120 289-296. (doi:10.1016/ j.ijcard.2006.11.221)

Chinda K, Sanit J, Chattipakorn S \& Chattipakorn N 2014 Dipeptidyl peptidase-4 inhibitor reduces infarct size and preserves cardiac function via mitochondrial protection in ischaemia-reperfusion rat heart. Diabetes \& Vascular Disease Research 11 75-83. (doi:10.1177/1479164113516134)

Cornoldi A, Caminiti G, Marazzi G, Vitale C, Patrizi R, Volterrani M, Miceli M, Fini M, Spera G \& Rosano G 2009 Effects of chronic testosterone administration on myocardial ischemia, lipid metabolism and insulin resistance in elderly male diabetic patients with coronary artery disease. International Journal of Cardiology 142 50-55. (doi:10.1016/j.ijcard.2008.12.107)

Culic V \& Busic Z 2015 Testosterone may influence left ventricular diastolic function depending on previous myocardial infarction and smoking. International Journal of Cardiology 186 67-71. (doi:10.1016/ j.ijcard.2015.03.238)

Curtis MJ, Hancox JC, Farkas A, Wainwright CL, Stables CL, Saint DA, Clements-Jewery H, Lambiase PD, Billman GE, Janse MJ et al. 2013 The Lambeth Conventions (II): guidelines for the study of animal and human ventricular and supraventricular arrhythmias. Pharmacology \& Therapeutics 139 213-248. (doi:10.1016/j.pharmthera.2013.04.008)

Ducimetiere P, Eschwege E, Papoz L, Richard J, Claude J \& Rosselin G 1980 Relationship of plasma insulin levels to the incidence of myocardial infarction and coronary heart disease mortality in a middle-aged population. Diabetologia 19 205-210.

Earnshaw WC, Martins LM \& Kaufmann SH 1999 Mammalian caspases: structure, activation, substrates, and functions during apoptosis. Annual Review of Biochemistry 68 383-424. (doi:10.1146/annurev. biochem.68.1.383)

El-Sherbeeny NA \& Nader MA 2016 The protective effect of vildagliptin in chronic experimental cyclosporine A-induced hepatotoxicity. Canadian Journal of Physiology and Pharmacology 94 251-256. (doi:10.1139/cjpp-2015-0336)

Espinola-Klein C, Gori T, Blankenberg S \& Munzel T 2011 Inflammatory markers and cardiovascular risk in the metabolic syndrome. Frontiers in Biosciences 16 1663-1774. (doi:10.2741/3812)

Flegal KM, Carroll MD, Kit BK \& Ogden CL 2012 Prevalence of obesity and trends in the distribution of body mass index among US adults, 1999-2010. JAMA 307 491-497. (doi:10.1001/jama.2012.39)

Foley PL 2005 Common surgical procedures in rodents. In Laboratory Animal Medicine and Management. Eds JD Reuter \& MA Suckow.

Published by Bioscientifica Ltd. 
Office of Animal Research Education and Compliance, University of Virginia, Charlottesville, VA, USA: International Veterinary Information Service, Ithaca, NY, USA. (www.ivis.org)

Francomano D, Bruzziches R, Barbaro G, Lenzi A \& Aversa A 2014 Effects of testosterone undecanoate replacement and withdrawal on cardio-metabolic, hormonal and body composition outcomes in severely obese hypogonadal men: a pilot study. Journal of Endocrinological Investigation 37 401-411. (doi:10.1007/s40618-014-0066-9)

Friedewald WT, Levy RI \& Fredrickson DS 1972 Estimation of the concentration of low-density lipoprotein cholesterol in plasma, without use of the preparative ultracentrifuge. Clinical Chemistry 18 499-502.

Garrido P, Salehzadeh F, Duque-Guimaraes DE \& Al-Khalili L 2014 Negative regulation of glucose metabolism in human myotubes by supraphysiological doses of 17 beta-estradiol or testosterone. Metabolism 63 1178-1187. (doi:10.1016/j.metabol.2014.06.003)

Giannocco G, Oliveira KC, Crajoinas RO, Venturini G, Salles TA, Fonseca-Alaniz MH, Maciel RM \& Girardi AC 2013 Dipeptidyl peptidase IV inhibition upregulates GLUT4 translocation and expression in heart and skeletal muscle of spontaneously hypertensive rats. European Journal of Pharmacology 698 74-86. (doi:10.1016/j.ejphar.2012.09.043)

Glorie LL, Verhulst A, Matheeussen V, Baerts L, Magielse J, Hermans N, D’Haese PC, De Meester I \& De Beuf A 2012 DPP4 inhibition improves functional outcome after renal ischemia-reperfusion injury. American Journal of Physiology: Renal Physiology 303 F681-F688. (doi:10.1152/ajprenal.00075.2012)

Halestrap AP \& Pasdois P 2009 The role of the mitochondrial permeability transition pore in heart disease. Biochimica et Biophysica Acta 1787 1402-1415. (doi:10.1016/j.bbabio.2008.12.017)

Handelsman DJ \& Zajac JD 2004 Androgen deficiency and replacement therapy in men. Medical Journal of Australia 180 529-535.

Hausenloy DJ, Duchen MR \& Yellon DM 2003 Inhibiting mitochondrial permeability transition pore opening at reperfusion protects against ischaemia-reperfusion injury. Cardiovascular Research 60 617-625. (doi:10.1016/j.cardiores.2003.09.025)

Huang C, Gu H, Zhang W, Herrmann JL \& Wang M 2010 Testosteronedown-regulated Akt pathway during cardiac ischemia/reperfusion: a mechanism involving BAD, Bcl-2 and FOXO3a. Journal of Surgical Research 164 e1-e11. (doi:10.1016/j.jss.2010.07.041)

Huisamen B, Genis A, Marais E \& Lochner A 2011 Pre-treatment with a DPP-4 inhibitor is infarct sparing in hearts from obese, pre-diabetic rats. Cardiovascular Drugs Therapy 25 13-20. (doi:10.1007/s10557-0106271-7)

Hwang TI, Liao TL, Lin JF, Lin YC, Lee SY, Lai YC \& Kao SH 2011 Low-dose testosterone treatment decreases oxidative damage in TM3 Leydig cells. Asian Journal of Andrology 13 432-437. (doi:10.1038/ aja.2010.159)

Ishii M, Shibata R, Kondo K, Kambara T, Shimizu Y, Tanigawa T, Bando YK, Nishimura M, Ouchi N \& Murohara T 2014 Vildagliptin stimulates endothelial cell network formation and ischemia-induced revascularization via an endothelial nitric-oxide synthase-dependent mechanism. Journal of Biological Chemistry 289 27235-27245. (doi:10.1074/jbc.M114.557835)

Kelly DM \& Jones TH 2013 Testosterone: a vascular hormone in health and disease. Journal of Endocrinology 217 R47-R71. (doi:10.1530/ joe-12-0582)

Krishnamoorthy A, Greiner MA, Bertoni AG, Eapen ZJ, O’Brien EC, Curtis LH, Hernandez AF \& Mentz RJ 2016 The obesity and heart failure epidemics among African Americans: insights from the Jackson Heart Study. Journal of Cardiac Failure 22 589-597. (doi:10.1016/j.cardfail.2016.03.004)

Lateef A, Khan AQ, Tahir M, Khan R, Rehman MU, Ali F, Hamiza OO \& Sultana S 2013 Androgen deprivation by flutamide modulates uPAR, MMP-9 expressions, lipid profile, and oxidative stress: amelioration by daidzein. Molecular and Cellular Biochemistry 374 49-59. (doi:10.1007/s11010-012-1504-7)

Lu MC, Tzang BS, Kuo WW, Wu FL, Chen YS, Tsai CH, Huang CY \& Lee SD 2007 More activated cardiac mitochondrial-dependent apoptotic pathway in obese Zucker rats. Obesity 15 2634-2642. (doi:10.1038/oby.2007.315)

Lu SF, Huang Y, Wang N, Shen WX, Fu SP, Li Q, Yu ML, Liu WX, Chen X, Jing XY et al. 2016 Cardioprotective effect of electroacupuncture pretreatment on myocardial ischemia/reperfusion injury via antiapoptotic signaling. Evidence-Based Complementary and Alternative Medicine 2016 4609784. (doi:10.1155/2016/4609784)

Mancini A, Leone E, Festa R, Grande G, Silvestrini A, de Marinis L, Pontecorvi A, Maira G, Littarru GP \& Meucci E 2008 Effects of testosterone on antioxidant systems in male secondary hypogonadism. Journal of Andrology 29 622-629. (doi:10.2164/ jandrol.107.004838)

Maruyama D, Hirata N, Tokinaga Y, Kawaguchi R \& Yamakage M 2016 Nitrite reduces ischemia-induced ventricular arrhythmias by attenuating connexin 43 dephosphorylation in rats. Anesthesia and Analgesia 122 410-417. (doi:10.1213/ANE.0000000000001063)

Ogden CL, Carroll MD, Fryar CD \& Flegal KM 2015 Prevalence of obesity among adults and youth: United States, 2011-2014. NCHS Data Brief $1-8$.

Peterson QP, Hsu DC, Goode DR, Novotny CJ, Totten RK \& Hergenrother PJ 2009 Procaspase-3 activation as an anti-cancer strategy: structure-activity relationship of procaspase-activating compound 1 (PAC-1) and its cellular co-localization with caspase-3. Journal of Medicinal Chemistry 52 5721-5731. (doi:10.1021/ jm900722z)

Pintana H, Pongkan W, Pratchayasakul W, Chattipakorn N \& Chattipakorn SC 2015 Testosterone replacement attenuates cognitive decline in testosterone-deprived lean rats, but not in obese rats, by mitigating brain oxidative stress. Age 37 84. (doi:10.1007/s11357-0159827-4)

Pongkan W, Chattipakorn SC \& Chattipakorn N 2015 Chronic testosterone replacement exerts cardioprotection against cardiac ischemia-reperfusion injury by attenuating mitochondrial dysfunction in testosterone-deprived rats. PLOS ONE 10 e0122503. (doi:10.1371/journal.pone.0122503)

Pongkan W, Pintana H, Sivasinprasan S, Jaiwongkam T, Chattipakorn N \& Chattipakorn SC 2016 Testosterone deprivation accelerates cardiac dysfunction in obese male rats. Journal of Endocrinology 229 209-220. (doi:10.1530/JOE-16-0002)

Pratchayasakul W, Kerdphoo S, Petsophonsakul P, Pongchaidecha A, Chattipakorn N \& Chattipakorn SC 2011 Effects of high-fat diet on insulin receptor function in rat hippocampus and the level of neuronal corticosterone. Life Science 88 619-627. (doi:10.1016/ j.lfs.2011.02.003)

Reckelhoff JF, Yanes LL, Iliescu R, Fortepiani LA \& Granger JP 2005 Testosterone supplementation in aging men and women: possible impact on cardiovascular-renal disease. American Journal of Physiology: Renal Physiology 289 F941-F948. (doi:10.1152/ ajprenal.00034.2005)

Rottman JN, Ni G \& Brown M 2007 Echocardiographic evaluation of ventricular function in mice. Echocardiography 24 83-89. (doi:10.1111/j.1540-8175.2006.00356.x)

Traish AM \& Kypreos KE 2011 Testosterone and cardiovascular disease: an old idea with modern clinical implications. Atherosclerosis 214 244-248. (doi:10.1016/j.atherosclerosis.2010.08.078)

Traish AM, Saad F \& Guay A 2009 The dark side of testosterone deficiency: II. Type 2 diabetes and insulin resistance. Journal of Andrology 30 23-32. (doi:10.2164/jandrol.108.005751)

Traish AM, Haider A, Doros G \& Saad F 2014 Long-term testosterone therapy in hypogonadal men ameliorates elements of the metabolic http://joe.endocrinology-journals.org

DOI: $10.1530 / J O E-16-0232$
() 2016 Society for Endocrinology Printed in Great Britain 
syndrome: an observational, long-term registry study. International Journal of Clinical Practice 68 314-329. (doi:10.1111/ijcp.12319)

Valle MM, Graciano MF, Lopes de Oliveira ER, Camporez JP, Akamine EH, Carvalho CR, Curi R \& Carpinelli AR 2011 Alterations of NADPH oxidase activity in rat pancreatic islets induced by a high-fat diet. Pancreas 40 390-395. (doi:10.1097/MPA.0b013e31820569d0)

Walters J, Pop C, Scott FL, Drag M, Swartz P, Mattos C, Salvesen GS \& Clark AC 2009 A constitutively active and uninhibitable caspase-3 zymogen efficiently induces apoptosis. Biochemical Journal 424 335-345. (doi:10.1042/BJ20090825)

Wu FC \& von Eckardstein A 2003 Androgens and coronary artery disease. Endocrine Reviews 24 183-217. (doi:10.1210/er.2001-0025)

Zhang L, Lei D, Zhu GP, Hong L \& Wu SZ 2013 Physiological testosterone retards cardiomyocyte aging in Tfm mice via androgen receptorindependent pathway. Chinese Medical Sciences Journal 28 88-94. (doi:10.1016/S1001-9294(13)60028-0)

Received in final form 16 August 2016

Accepted 19 August 2016

Accepted Preprint published online 19 August 2016 (c) 2016 Society for Endocrinology Printed in Great Britain
Published by Bioscientifica Ltd 